\title{
Measurement of Coronary Bifurcation Angle with Coronary CT Angiography: A Phantom Study
}

\author{
Sogol Givehchi ${ }^{\mathrm{a}}$, Mohammad Javad Safari ${ }^{\mathrm{a}, \mathrm{b}}$, Sock Keow Tan ${ }^{\mathrm{a}}$, Mohammad Nazri \\ Bin Md Shaha, ${ }^{\mathrm{a} b}$, Fadhli Bin Mohamed Sani ${ }^{\mathrm{a}, \mathrm{b}}$, Raja Rizal Azman Bin Raja Amanª, \\ Zhonghua Sunc, Chai Hong Yeonga, ${ }^{\mathrm{a}, \mathrm{b}}$, Kwan Hoong Ng ${ }^{\mathrm{a}, \mathrm{b}}$, and Jeannie Hsiu Ding \\ Wong $^{\mathrm{a}, \mathrm{b}, *}$
}

${ }^{a}$ Department of Biomedical Imaging, Faculty of Medicine, University of Malaya, Kuala Lumpur 50603, Malaysia

${ }^{\mathrm{b}}$ University of Malaya Research Imaging Centre (UMRIC), Faculty of Medicine, University of Malaya, Kuala Lumpur 50603, Malaysia

${ }^{\mathrm{c}}$ Department of Medical Radiation Sciences, Curtin University, Perth, 6845, Australia

Address correspondence to: Dr. Jeannie Hsiu Ding Wong,

Department of Biomedical Imaging, Faculty of Medicine, University of Malaya, Kuala Lumpur 50603, Malaysia.

Tel. No. (Office): 03-79493359

E-mail Address: jeannie wong80@um.edu.my 
Purpose: Accurate determination of the bifurcation angle and correlation with plaque buildup may lead to the prediction of coronary artery disease (CAD). This work evaluates two techniques to measure bifurcation angles in 3D space using coronary computed tomography angiography (CCTA).

Materials and Methods: Nine phantoms were fabricated with different bifurcation angles ranging from $55.3^{\circ}$ to $134.5^{\circ}$. General X-ray and CCTA were employed to acquire 2D and 3D images of the bifurcation phantoms, respectively. Multiplanar reformation (MPR) and volume rendering technique (VRT) were used to measure the bifurcation angle between the left anterior descending (LAD) and left circumflex arteries (LCx). The measured angles were compared with the true values to determine the accuracy of each measurement technique. Interobserver variability was evaluated. The two techniques were further applied on 50 clinical CCTA cases to verify its clinical value.

Results: In the phantom setting, the mean absolute differences calculated between the true and measured angles by MPR and VRT were $2.4^{\circ} \pm 2.2^{\circ}$ and $3.8^{\circ} \pm 2.9^{\circ}$, respectively. Strong correlation was found between the true and measured bifurcation angles. Furthermore, no significant differences were found between the bifurcation angles measured using either technique. In clinical settings, large difference of $12.0^{\circ} \pm 10.6^{\circ}$ was found between the two techniques.

Conclusion: In the phantom setting, both techniques demonstrated a significant correlation to the true bifurcation angle. Despite the lack of agreement of the two techniques in the clinical context, our findings in phantoms suggest that MPR should be preferred to VRT for the measurement of coronary bifurcation angle by CCTA. 
Keywords: Atherosclerosis, Coronary artery disease, Bifurcation angle, Coronary CT angiography

\section{'Introduction}

The coronary bifurcation angle has been related to the development of coronary artery disease (CAD). Coronary plaques were observed to distribute mostly near the side branches of coronary arteries, where the flow is non-uniform, and at the lesser curvature of bends where blood flow speeds are relatively low [1, 2]. A number of studies have identified coronary bifurcation angle as an important anatomical characteristic for the diagnosis and treatment of CAD [3-7]. The bifurcation angle formed by two main coronary branches, the left anterior descending (LAD) and left circumflex (LCx) arteries has been shown to be of particular importance [8-12]. Larger bifurcation angles were found to correlate with the growth of plaque buildup and is proposed as a biomarker for predicting the risk of CAD [13]. Accurate assessment of the bifurcation angle is also essential in design optimization and implementation of coronary stents, which consequently reduces restenosis and stent thrombosis, leading to improved outcome for patients undergoing interventional treatment of the atherosclerosis [14-17].

Coronary computed tomography angiography (CCTA) as a method for assessing the coronary arteries provides advantages over conventional angiography. Being less invasive in nature, it has fewer procedure-related complications and faster acquisition time. It also requires less amount of contrast medium $[13,18,19]$. This technique has been found to be highly accurate in diagnosing $\mathrm{CAD}$ due to its improved spatial and temporal resolution.

Advancements in CT technology has enabled a variety of 2D and 3D image rendering and processing techniques, such as multiplanar reformation (MPR), volume-rendering techniques 
(VRT), maximum intensity projections (MIP), minimum intensity projections (MINIP), and shaded surface display (SSD) [20]. A number of studies have utilized some of these postprocessing techniques in determining the bifurcation angle $[12,13,18,21]$. Sun and his colleagues used the VRT technique to measure the left coronary bifurcation angle [18, 21]. Mieghem et al. applied MPR on the CCTA images to obtain the bifurcation angles [22]. Cui et al further confirmed the clinical value of using bifurcation angle for prediction of significant coronary stenosis [23]. Juan et al in their recent study reported the feasibility of using 2D axial images for measurement of left bifurcation angle [24]. However, accuracy of these measurement methods has not been fully determined. Therefore, the purpose of this study is to evaluate the accuracy of two techniques (MPR and VRT) to measure the left bifurcation angle, based on CCTA images of a custom-made heart phantom. The two techniques were further tested on 50 clinical cases to evaluate the comparability of the two techniques in real case scenarios.

\section{Materials and Methods}

\section{A. Fabrication of the phantoms}

A heart-shaped phantom attached with a bifurcation angle phantom (BAP) was constructed to model the bifurcation angle between the LAD and LCx (Figure 1). For the bifurcation angle phantom, a $4.17 \mathrm{~mm}$ diameter urine drainage tube was used to model the LAD artery while a $3.19 \mathrm{~mm}$ diameter catheter was used to model the LCx artery (Figure 1a). The two tubes were attached together by using a V-shape metal rod (1.0 mm in diameter) that was inserted into the urine tube via a $3.0 \mathrm{~mm}$ hole. The V-shape metal rod served to fixate the bifurcation angle. The hole was subsequently sealed. The BAP was filled with a contrast agent (Ultravist- 370, 50ml) 
and sealed at all ends. A total of nine BAPs (ranging from $50^{\circ}$ to $130^{\circ}$ ) were created by changing the angulation of the v-shaped rods. Whilst the V-shape rods were able to fixate the angulation between the urine tube and catheter at a fixed position, the actual resulting bifurcation angle was determined using 2D X-ray images of the BAP. The BAPs were placed on top of polystyrene foam and X-ray images were taken using a digital radiography unit (DRXEvolution, Carestream Health Inc., Toronto, Canada).

In order to mimic the left coronary bifurcation angle sitting on the heart, representing a more realistic cardiac situation, the BAP was mounted on a heart-shaped phantom. The heart-shaped phantom was fabricated using polyacrylamide, contains acrylamide monomer (AA; gelling agent), N, N0-methylene-bis-acrylamide (MBA) (crosslinking agent), NaCl, N, N, N0, N0tetra-metyl-ethylenediamine (TEMED) (catalyser), and ammonium persulfate (APS) (polymerisation primer), all mixed in degassed water. The polyacrylamide was modeled into a heart-shape and left to set for a few minutes. After that, it was sealed with a thin plastic sheet to avoid water evaporation (Figure 1b). 


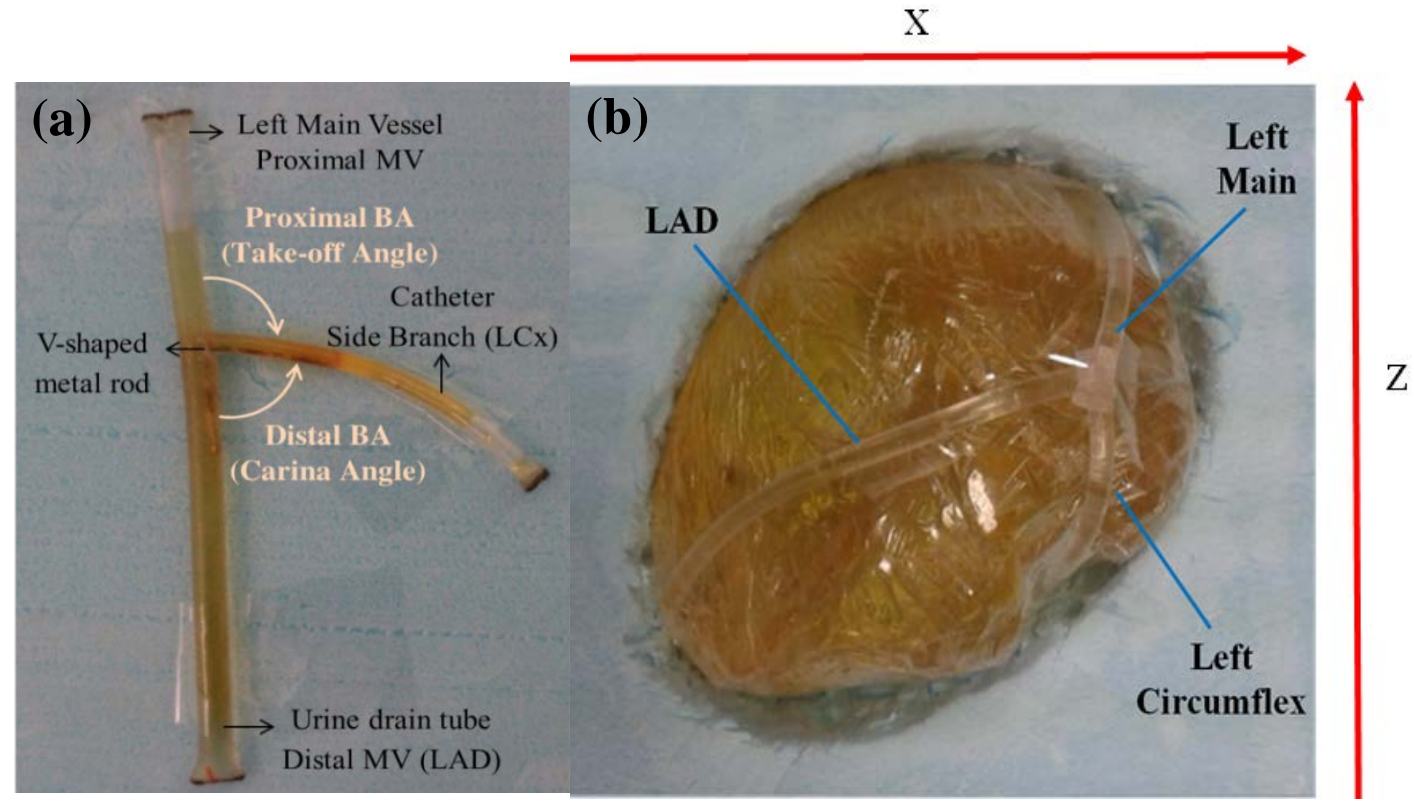

Figure 1 (a) A simple phantom containing the bifurcation angle between LAD and LCx. (b) Bifurcation angle phantom mounted on the heart-shaped phantom. The $\mathrm{X}$ and $\mathrm{Z}$ axes show the position of the BAHP with respect to the CT scanner frame of reference.

\section{B. Measurement of the true bifurcation angle}

The true bifurcation angle was determined using 2D x-ray images. Digital imaging and communications in medicine (DICOM) images were transferred to DICOM Viewer software (RadiAnt DICOM VIEWER, Medixant, Poznan, Poland) and the inner and outer angles of the nine BAPs were measured three times and averaged (Figure 2). 


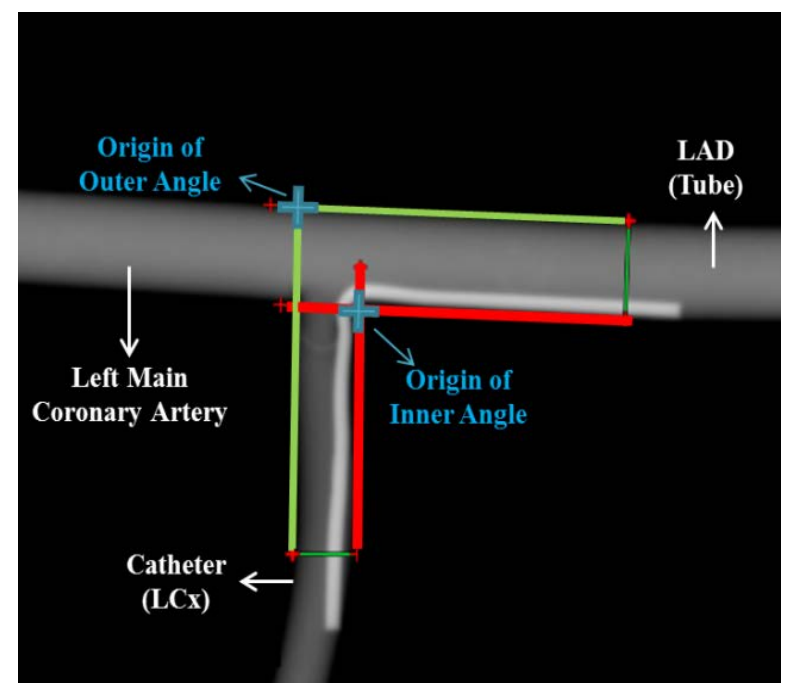

Figure 2 Planar X-ray image of the BAP.

\section{Measurement of bifurcation angles in three-dimensional space using CCTA}

The bifurcation angle heart phantom (BAHP) was scanned using a 64-slice CT scanner (Somatom Siemens, Erlangen, Germany). The scan parameters were as follows: collimator detector of $64 \times 0.6 \mathrm{~mm}^{2}$, gantry rotation time of $0.33 \mathrm{~s}$, with tube voltage of $120 \mathrm{kVp}$ and tube current modulation ranging from 55 to 320 mAs. The BAHP was placed with the left main and left circumflex vessels aligned along the X-axis of the CT scanner frame of reference (Figure 1b). Transaxial images were reconstructed with $0.75 \mathrm{~mm}$ slice thickness and $0.5 \mathrm{~mm}$ increments. The geometric measurements were performed using Advantage Workstation Server (version 3.0, GE Healthcare, Chicago, Illinois). Two radiologists (with more than 5 years of experience) evaluated the bifurcation angles separately using two different techniques, (i) MPR and (ii) VRT technique, respectively. A third radiologist (also with more than 5 years of experience) was asked to measure the bifurcation angles using both techniques to evaluate the inter-observer variation and the dependency of the techniques on operators. The radiologists were asked to measure both the inner and outer angles of the nine BAHPs. 
For the MPR technique, the images were viewed on thin $0.75 \mathrm{~mm}$ slices in MPR views. Firstly the coronal plane (1) was aligned to the long axis of the LM perpendicular to the plane of bifurcation (2) (Figure 3a). The short axis plane (3) was then adjusted to show the LAD and LCx in the best transverse view (showing both vessels end-on and approximately equal in size). Finally, the imaging plane parallel to the plane of bifurcation (2) was adjusted to transect the center of both LAD and LCx at 1-cm away from the bifurcation point. The resulting adjusted view should then show the LM, LAD, and LCx with the largest LAD/LCx angle possible (Figure 3b). The inner and outer angles of the LAD-LCx were then measured in this plane, up till 1-cm from the point of bifurcation (Figure 3b).

For the VRT technique, a 3D volume rendering of the BAHP was performed. The phantom was then rotated to find an optimal view (i.e. perpendicular view to the plane of bifurcation) of the bifurcation angle and centered at the LAD/LCx bifurcation. The inner and outer angles were then measured and averaged (Figure 4).
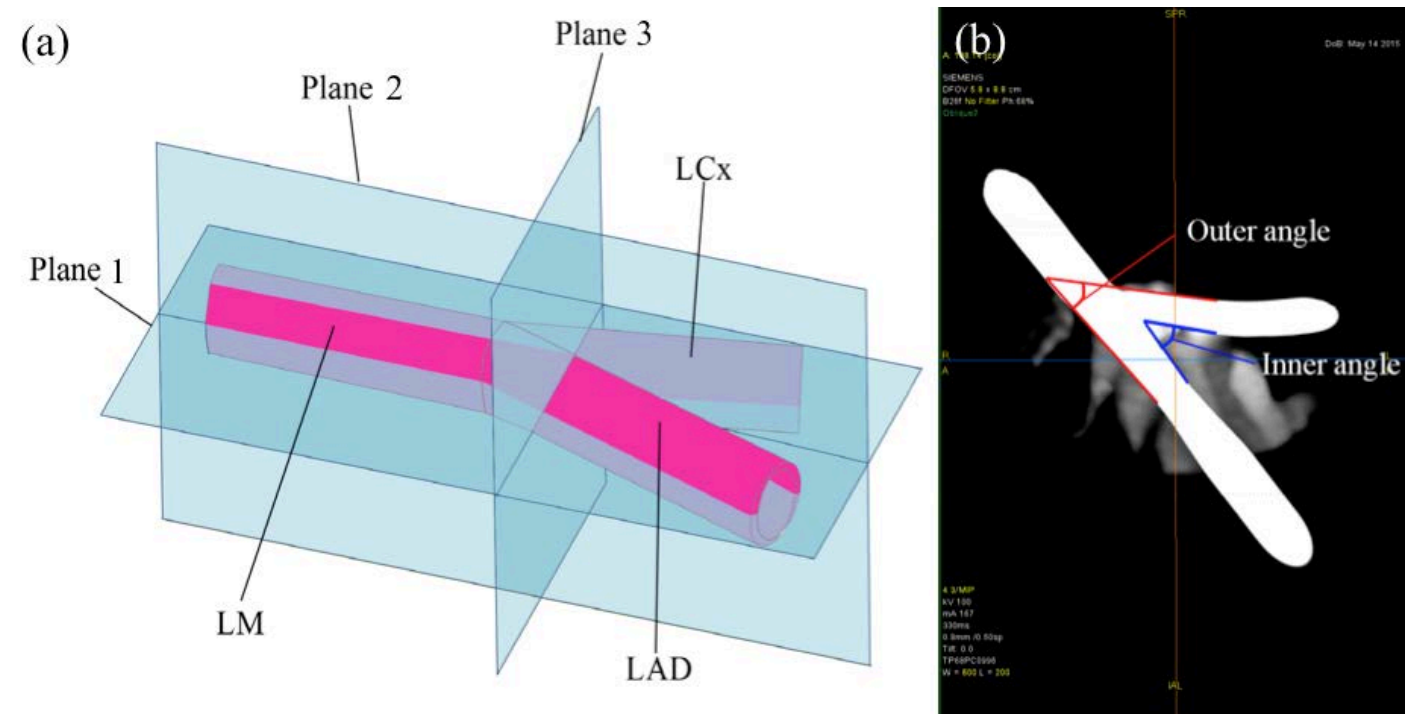

Figure 3 (a) The MPR technique’s imaging planes (1, 2, and 3) with resulting inner and outer bifurcation angles (b). 


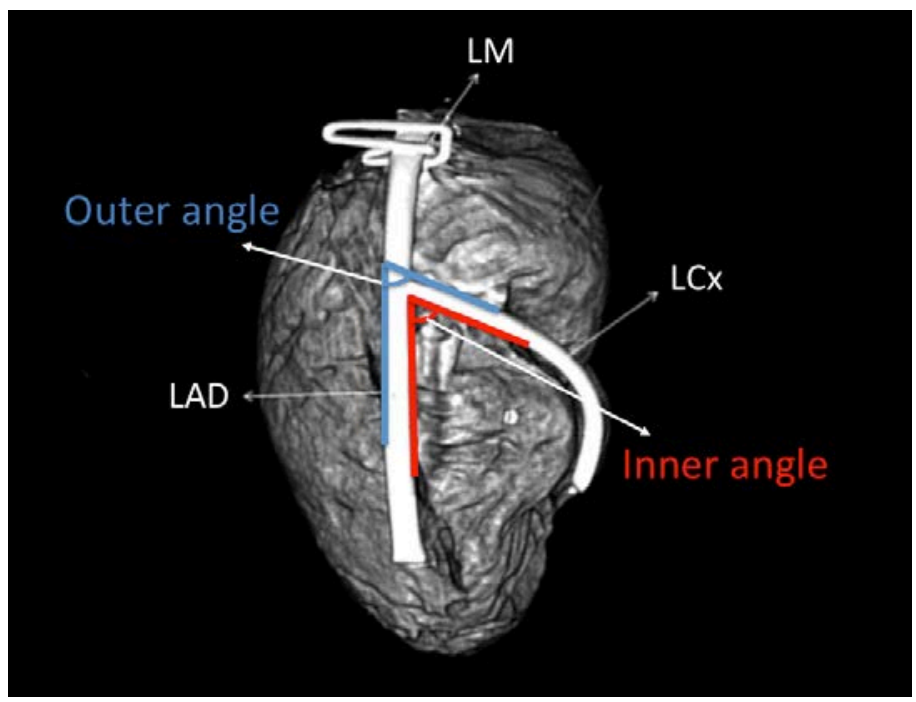

Figure 4 The VRT technique to measure the outer and the inner bifurcation angle between LAD and LCx.

\section{Measurement of bifurcation angles of clinical cases}

In order to study the correlation of the MPR and VRT techniques in the clinical context, the bifurcation angle of 50 clinical cases that underwent CCTA was investigated. The same radiologists were asked to measure and report the inner and outer angles of patient bifurcation angles using MPR and VRT techniques.

\section{E. Statistical Analysis}

Statistical analysis was performed using IBM SPSS Statistics, version 22.0 (IBM Corp., Armonk, NY) and MedCalc ${ }^{\circledR}$, version 16.2.1, (MedCalc Software bvba, Ostend, Belgium). The mean absolute difference between bifurcation angle phantom true angles and measured angles by each technique was calculated. Wilcoxon signed ranks tests were carried out to determine whether there is any significant difference between two measurements. A $p$ value of less than 0.05 was defined as statistically significant. Bland-Altman plots were used to assess the agreement between measured values using both techniques compared to the phantom true 
angles, as well as the agreement between both techniques, and inter-observer measured bifurcation angle.

\section{Results and Discussions}

\section{A. Phantom study}

The mean absolute differences between measured and the true values of the bifurcation angles were $2.4^{\circ} \pm 2.2^{\circ}$ and $3.8^{\circ} \pm 2.9^{\circ}$ for MPR and VRT techniques, respectively. The mean absolute difference between the MPR and VRT measured bifurcation angles was $4.0^{\circ} \pm 3.0^{\circ}$.

No significant differences were found between both techniques and also between the MPR and VRT measured angles with the true angles. Strong and significant correlation was found between the true angles with the MPR $(r=0.98, p<0.05)$ and VRT $(r=0.99, p<0.05)$ measured angles (Figure 5).

Bland-Altman plots were constructed to evaluate the degree of agreement between these two measurement techniques, compared to the true bifurcation angle (Figure 6). The MPR technique tends to over-predict the bifurcation angle by $0.4^{\circ}$ while the VRT technique tends to underpredict the bifurcation angle by $0.7^{\circ}$. The MPR technique shows a slightly narrower 95\% confidence interval (CI) limit to agreement $\left(-5.9^{\circ}-6.8^{\circ}\right)$ compared to the VRT technique ($10.2^{\circ}-8.8^{\circ}$ ). This indicates that the MPR technique may be more accurate compared to the VRT technique.

Inter-observer variability was carried out in order to clarify the dependency of each technique on operator's performance. Bland-Atman plots in Figure 7 shows that the mean differences in the bifurcation angles measured by two different observers were $2^{\circ}$ and $0.4^{\circ}$ for MPR and VRT techniques, respectively. The MPR technique again shows a slightly narrower $95 \%$ CI of $\left(-10.1^{\circ}\right.$ 
$\left.-6.2^{\circ}\right)$ compared to VRT technique $\left(-11.3^{\circ}-12.0^{\circ}\right)$. The narrower limit to agreement of the MPR technique shows that the MPR technique is less dependent on operators.

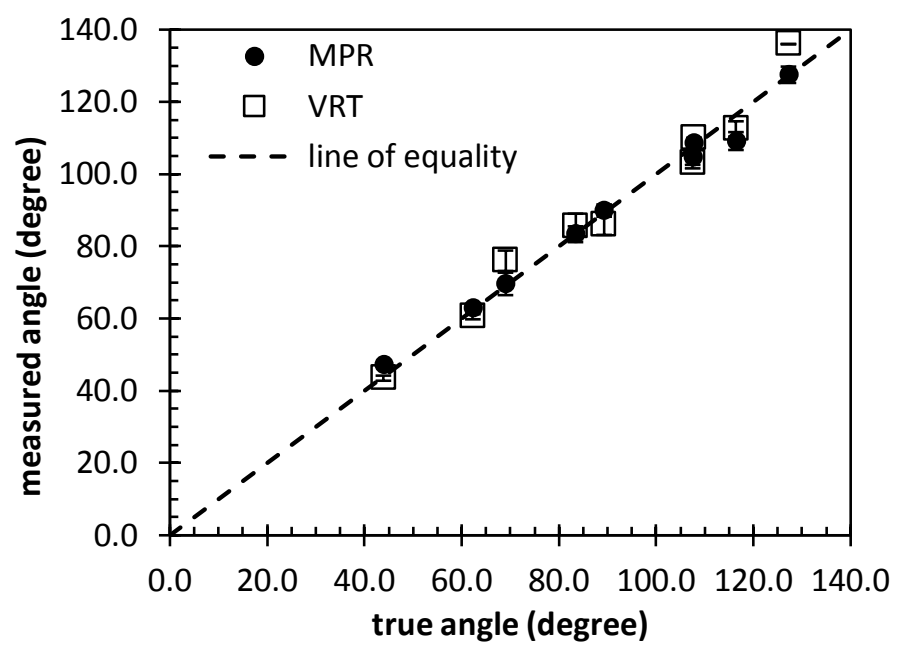

Figure 5 Scatter plot of the bifurcation angles measured using MPR and VRT techniques. Error bars represent the mean error of the inner and outer angle measurements.

(a)

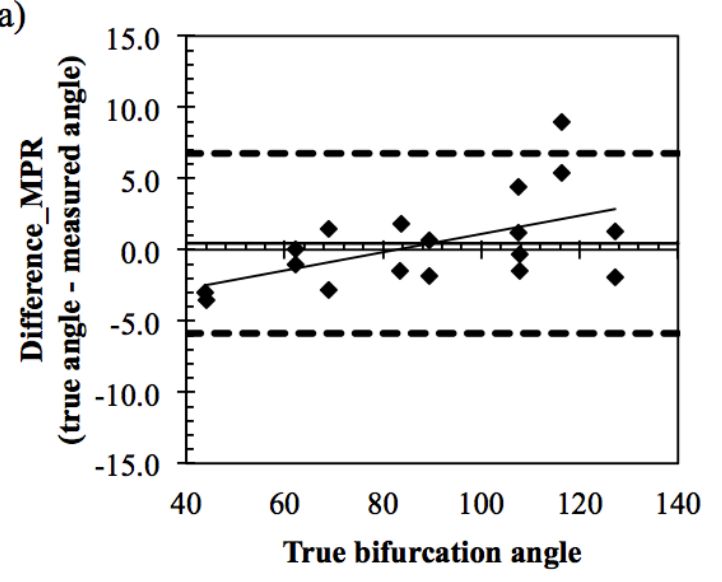

$---+1.96 \mathrm{~s}$

$---1.96 \mathrm{~s}$

Mean

- MPR

Linear (MPR) (b)

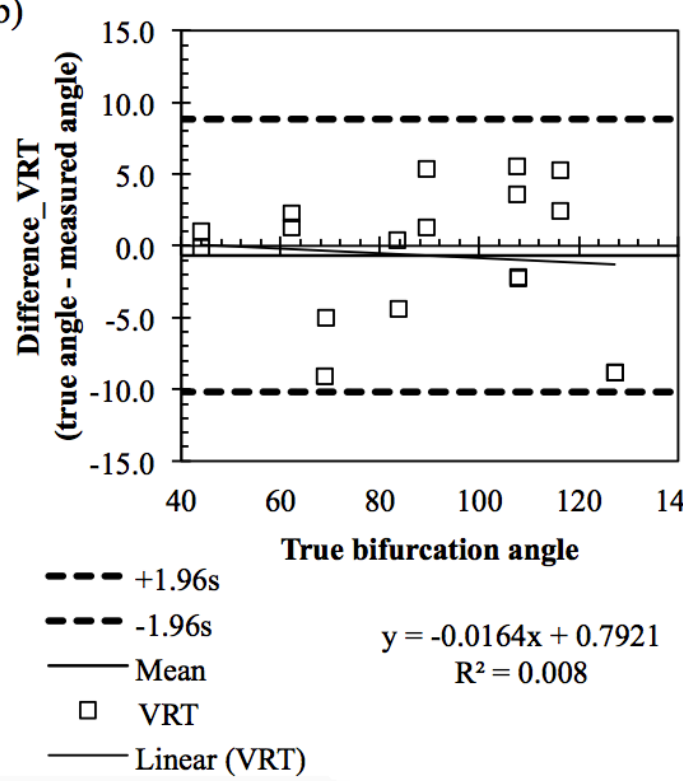

Figure 6 Bland-Altman plots comparing the difference between the true and measured bifurcation angles using (a) MPR and (b) VRT techniques. 
(a)

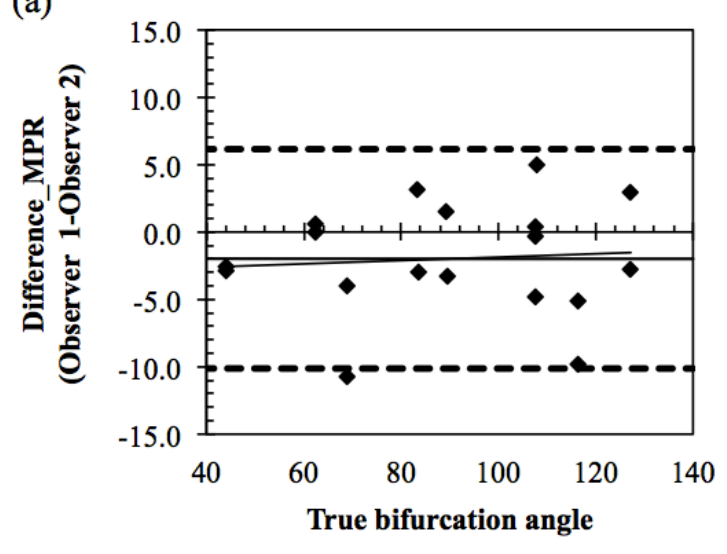

\begin{tabular}{l}
$--+1.96 \mathrm{~s}$ \\
$---1.96 \mathrm{~s}$ \\
\hline Mean \\
$-\quad$ MPR \\
- Linear (MPR)
\end{tabular} (b)
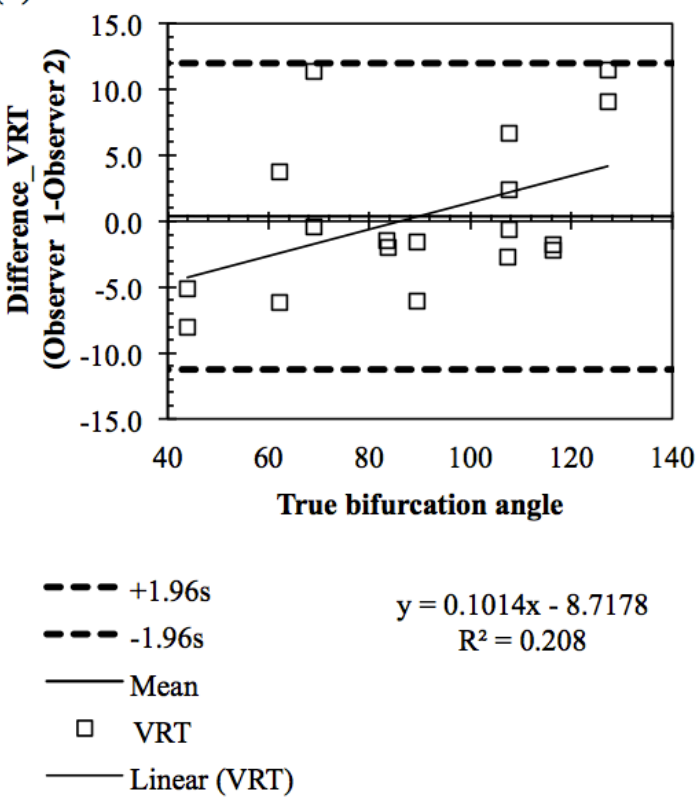

Figure 7 Bland-Altman plots for inter-observer measured bifurcation angles by (a) MPR and

(b) VRT techniques as compared to the true bifurcation angle.

\section{B. Clinical measurement}

Fifty clinical CCTA cases were included in the study, of which $52 \%$ were female and $48 \%$ were male patients. The mean absolute difference between the inner and outer bifurcation angles were $10.9^{\circ} \pm 12.7^{\circ}$ and $7.6^{\circ} \pm 11.0^{\circ}$, for MPR and VRT measurements, respectively. The large discrepancies between the inner and outer bifurcation angles measured in the clinical data are due to the variation in the diameter of the blood vessels in patients within the 1-cm distance from the bifurcation site, in contrast with the uniform diameter of the tubes used in the phantom. There were no significant differences in the bifurcation angles measured using MPR and VRT. Significant correlation was also found between the angles measured using both techniques $(\mathrm{r}=$ 0.85). 
The mean absolute difference between the MPR and VRT measurements was $12.0^{\circ} \pm 10.6^{\circ}$. Compared to the phantom study, the mean absolute difference observed between the two techniques is $8.0^{\circ}$ higher in the clinical data. This may be due to the variation in the anatomy of the heart and blood vessels and tortuosity of the blood vessels in the patients. Bland-Altman plot was constructed to evaluate the agreement between MPR and VRT technique (Figure 8). MPR over-estimated the bifurcation angles by $0.3^{\circ}$. $95 \%$ of the differences between MPR and VRT would be within $\pm 31.7^{\circ}$. This shows a poor agreement between the two techniques.

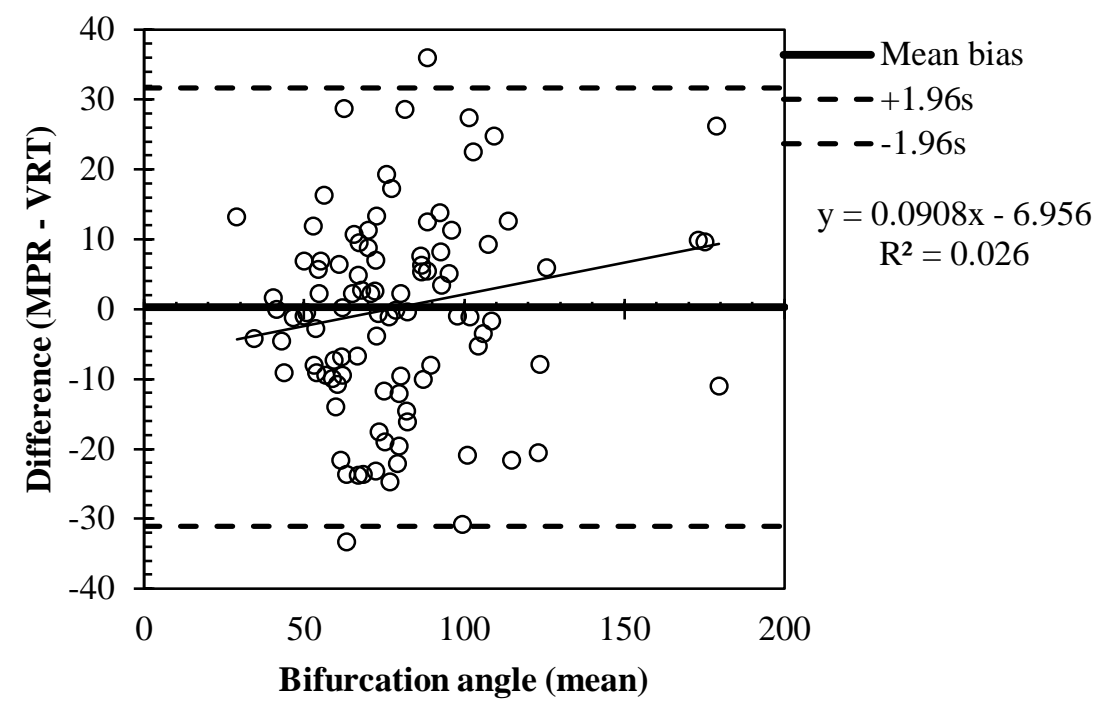

Figure 8 Bland-Altman plot showing the difference in the bifurcation angles measured by MPR and VRT techniques.

There was no significant correlation between the differences in the bifurcation angles measured by either technique with the degree of the bifurcation angles $(r=0.15)$. In other words, a larger coronary bifurcation angle does not indicate larger differences measured using MPR or VRT technique. 
In the phantom study, the mean absolute differences of both the MPR and VRT techniques discussed here is higher than the technique used by Craiem et al., whereby they reported a mean absolute difference of less than $0.5^{\circ}$ using their automated technique [12]. This is expected as both the MPR and VRT techniques require the manual delineation of angles by the radiologist and therefore is subjected to higher uncertainty. However, in the absence of automated software to measure the bifurcation angle accurately, MPR and VRT techniques are tools that most radiologists are familiar with and are available in most image processing software installed at the CT console or reporting area. Between the two techniques, MPR showed narrower limits to agreement when compared to the true bifurcation angles and less dependant on operator in the phantom study, which means that this technique would show slightly higher accuracy compared to VRT technique. The MPR view contains three perpendicular planes and corresponding 3D oblique plane that allows a radiologist to move in a precise manner and find the exact location of the bifurcation; while the manual rotation in VRT to find the best orthogonal view of the bifurcation angle via 'eye-balling' is a subjective decision by the radiologist. Further, Juan et al reported that 2D axial images are also reliable in measuring the left bifurcation angle when compared to invasive coronary angiography [24]. Their results support our findings of using 2D reconstructions for clinical measurements of bifurcation angles.

The MPR and VRT techniques showed poor agreement in the clinical application. This is not surprising, as anatomical variation of the blood vessels would exacerbate the subjective evaluation by the radiologists, particularly the VRT technique. While it was not possible to know which technique is more accurate based on the clinical measurements solely, because it is difficult to verify the true coronary bifurcation angle of each patient, the phantom study was useful to show that the MPR technique was more accurate compared to the VRT technique. 
A recent study has further confirmed the correlation between left coronary bifurcation angle with the development of coronary artery disease, in particular, male patients with large body mass index have been shown to be highly associated with larger bifurcation angle, indicating a higher risk of CAD [25]. Our study aims to improve accuracy in measurement of the bifurcation angle and to provide evidence for the optimal technique once angle measurement is incorporated into the diagnostic analysis of coronary artery disease as an important risk factor.

Several limitations in this study must be acknowledged. Firstly, straight tubes with constant diameters were used to fabricate the simple shape of the bifurcation site, whereas we are aware that coronary arteries are tortuous and the diameter of the arteries varies. To address this problem, all three measurement techniques (2D, MPR, and VRT) were measured within the first 1-cm from the inner bifurcation angle. When applying these measurement techniques in real patients, it is recommended that the measurement of the bifurcation angles is made close (within 1-cm) to the intersection of the two arteries. Secondly, movement of left main coronary bifurcation during the cardiac cycle is complex and consequently may change the distal and proximal bifurcation angles. This effect on the bifurcation angle was not investigated in this study. The BAHP in this work does not model the real-time motion effect of a real heart. Further studies based on a realistic cardiac phantom would be desirable to verify our findings. Finally, correlation of the degree of bifurcation angle with hemodynamic effects to coronary artery represents another future research direction with use of realistic cardiac phantom since coronary artery disease is associated with angulation of the left coronary artery [2, 26].

\section{Conclusion}

We evaluate the accuracy of two different techniques, namely the MPR and VRT techniques, of measuring coronary bifurcation angles in 3D space based on CCTA images using a custom- 
made bifurcation angle heart phantom. The two techniques were used and validated in 50 clinical CCTA cases. The findings of this study show that both techniques demonstrated significant correlations to the true bifurcation angles. When used on clinical data, large differences were found between the bifurcation angles measured by the two techniques. Based on the phantom study, MPR technique was found to be more accurate and less sensitive to operator subjectivity.

\section{Acknowledgement}

The authors acknowledge and thank Dr. Ali Dabbagh at the Faculty of Medicine, University of Malaya for his support in this study. This study was supported by the High Impact Research (HIR) (grant number UM.C/625/1/HIR/MOHE/MED/36); and PPP from the University of Malaya (grant number PO015-2015B \& PO029-2015B).

\section{References}

[1] Davies PF, Polacek DC, Shi C, Helmke BP. The convergence of haemodynamics, genomics, and endothelial structure in studies of the focal origin of atherosclerosis. Biorheology. 2002;39:299-306.

[2] Chaichana T, Sun Z, Jewkes J. Computation of hemodynamics in the left coronary artery with variable angulations. J Biomech. 2011;44:1869-78.

[3] Vassilev D, Gil RJ. Relative dependence of diameters of branches in coronary bifurcations after stent implantation in main vessel--importance of carina position. Kardiologia polska. 2008;66:371-8; discussion 9.

[4] Ormiston JA, Webster MW, El Jack S, Ruygrok PN, Stewart JT, Scott D, et al. Drugeluting stents for coronary bifurcations: Bench testing of provisional side- branch strategies. Catheter Cardio Inte. 2006;67:49-55. 
[5] Legrand V, Thomas M, Zelisko M, De Bruyne B, Reifart N, Steigen T, et al. Percutaneous coronary intervention of bifurcation lesions: state-of-the-art. Insights from the second meeting of the European Bifurcation Club. EuroIntervention. 2007;3:44-9.

[6] Birim Ö, van Gameren M, Bogers AJ, Serruys PW, Mohr FW, Kappetein AP. Complexity of coronary vasculature predicts outcome of surgery for left main disease. The Annals of thoracic surgery. 2009;87:1097-105.

[7] Collins N, Seidelin PH, Daly P, Ivanov J, Barolet A, Mackie K, et al. Long-term outcomes after percutaneous coronary intervention of bifurcation narrowings. Am J Cardiol. 2008;102:404-10.

[8] Rubinshtein R, Lerman A, Spoon DB, Rihal CS. Anatomic features of the left main coronary artery and factors associated with its bifurcation angle: a 3-dimensional quantitative coronary angiographic study. Catheter Cardiovasc Interv. 2012;80:304-9.

[9] Davies PF. Hemodynamic shear stress and the endothelium in cardiovascular pathophysiology. Nat Clin Pract Cardiovasc Med. 2009;6:16-26.

[10] Soulis JV, Farmakis TM, Giannoglou GD, Louridas GE. Wall shear stress in normal left coronary artery tree. J Biomech. 2006;39:742-9.

[11] Rodriguez-Granillo GA, Rosales MA, Degrossi E, Durbano I, Rodriguez AE. Multislice CT coronary angiography for the detection of burden, morphology and distribution of atherosclerotic plaques in the left main bifurcation. Int J Card Imaging. 2007;23:389-92.

[12] Craiem D, Casciaro ME, Graf S, Glaser CE, Gurfinkel EP, Armentano RL. Coronary arteries simplified with 3D cylinders to assess true bifurcation angles in atherosclerotic patients. Cardiovasc Eng. 2009;9:127-33.

[13] Sun Z, Cao Y. Multislice CT angiography assessment of left coronary artery: correlation between bifurcation angle and dimensions and development of coronary artery disease. Eur J Radiol. 2011;79:e90-5.

[14] Handran CB, Garberich RF, Lesser JR, Henry TD, Gilmore M, Schwartz RB. The Left Main Bifurcation Angle and Changes Throughout the Cardiac Cycle: Quantitative Implications for Left Main Bifurcation Stenting and Stents. J Invasive Cardiol. 2015;27:401-4.

[15] Lefevre T, Louvard Y, Morice MC, Loubeyre C, Piechaud JF, Dumas P. Stenting of bifurcation lesions: a rational approach. J Invasive Cardiol. 2001;14:573-85. 
[16] Pflederer T, Ludwig J, Ropers D, Daniel WG, Achenbach S. Measurement of coronary artery bifurcation angles by multidetector computed tomography. Invest. Radiol. 2006;41:793-8.

[17] Tu S, Jing J, Holm NR, Onsea K, Zhang T, Adriaenssens T, et al. In vivo assessment of bifurcation optimal viewing angles and bifurcation angles by three-dimensional (3D) quantitative coronary angiography. Int J Card Imaging. 2012;28:1617-25.

[18] Sun Z. Coronary CT angiography in coronary artery disease: correlation between virtual intravascular endoscopic appearances and left bifurcation angulation and coronary plaques. Biomed Res Int. 2013;2013:732059.

[19] Guo W, Liu X, Gao Z, Pirbhulal S, Huang W, Lin WH, et al. Quantification of threedimensional computed tomography angiography for evaluating coronary luminal stenosis using digital subtraction angiography as the standard of reference. Biomed Eng Online. 2015;14:50.

[20] Sabarudin A, Sun Z. Coronary CT angiography: Diagnostic value and clinical challenges. World J Cardiol. 2013;5:473-83.

[21] Xu L, Sun Z. Coronary CT angiography evaluation of calcified coronary plaques by measurement of left coronary bifurcation angle. Int J Cardiol. 2015;182:229-31.

[22] Van Mieghem CA, Thury A, Meijboom WB, Cademartiri F, Mollet NR, Weustink AC, et al. Detection and characterization of coronary bifurcation lesions with 64-slice computed tomography coronary angiography. Eur Heart J. 2007;28:1968-76.

[23] Cui Y, Zeng W, Yu J, Lu J, Hu Y, Diao N, et al. Quantification of left coronary bifurcation angles and plaques by coronary computed tomography angiography for prediction of significant coronary stenosis: A preliminary study with dual-source CT. PloS one. 2017;12:e0174352.

[24] Juan YH, Tsay PK, Shen WC, Yeh CS, Wen MS, Wan YL. Comparison of the Left Main Coronary Bifurcating Angle among Patients with Normal, Non-significantly and Significantly Stenosed Left Coronary Arteries. Scientific Reports. 2017;7.

[25] Temov K, Sun Z. Coronary computed tomography angiography investigation of the association between left main coronary artery bifurcation angle and risk factors of coronary artery disease. Int J Card Imaging. 2016. 
[26] Dong J, Sun Z, Inthavong K, Tu J. Fluid-structure interaction analysis of the left coronary artery with variable branch angulation. Comput Med Biomech Biomed Eng 2015; 18: 1500-1508.

[27] Dong J, Sun Z, Inthavong K, Tu J. Fluid-structure interaction analysis of the left coronary artery with variable angulation. Comput Med Biomech Biomed Eng 2015;18:1500-8. 